\title{
Assessment and repair of aortic valve cusp prolapse: Implications for valve-sparing procedures
}

\author{
Munir Boodhwani, MD, MMSc, ${ }^{\text {a,b }}$ Laurent de Kerchove, MD, ${ }^{\text {a }}$ Christine Watremez, MD, ${ }^{\text {a }}$ \\ David Glineur, MD, ${ }^{\mathrm{a}}$ Jean-Louis Vanoverschelde, MD, ${ }^{\mathrm{a}}$ Philippe Noirhomme, MD, ${ }^{\mathrm{a}}$ and \\ Gebrine El Khoury, MD
}

\begin{abstract}
Objectives: Cusp prolapse causing aortic insufficiency is associated with unique echocardiographic, clinical, and surgical features. Recognition and appropriate surgical repair of this pathologic condition can not only treat affected patients but also improve results of aortic valve-sparing procedures, for which pre-existing or induced cusp prolapse is an important cause of failure.
\end{abstract}

Methods: Of 428 patients undergoing aortic valve repair, 195 (46\%) were treated for cusp prolapse, and 111 $(57 \%)$ of those had trileaflet aortic valve and make up this cohort. Cusp disease was the sole mechanism for aortic insufficiency (isolated group) in 50 patients whereas aortic dilatation was contributory in 61 (associated group). In total, 144 cusps were repaired in 111 patients. Preoperative echocardiograms, intraoperative findings, and clinical and echocardiographic outcomes were reviewed.

Results: On preoperative echocardiography, presence of an eccentric aortic insufficiency jet, regardless of severity, had $92 \%$ sensitivity and $96 \%$ specificity for the detection of single cusp prolapse. A transverse fibrous band was characteristically identified on the prolapsing cusp (sensitivity $57 \%$; specificity $92 \%$ ), correctly localizing a prolapsing cusp in all cases. Freedom from aortic valve reoperation at 8 years was $100 \%$ in the isolated group and $93 \% \pm 5 \%$ in the associated group $(\mathrm{p}=0.33)$. Freedom from recurrent aortic insufficiency $(>2+)$ at 5 years was $90 \% \pm 5 \%$ in the isolated and $85 \% \pm 8 \%$ in the associated group $(P=.54)$. The choice of surgical technique did not affect aortic insufficiency recurrence at follow-up $(P=.6)$.

Conclusions: Recognition and repair of isolated aortic cusp prolapse provides durable midterm outcome. An eccentric aortic insufficiency jet and a fibrous band can aid in the diagnosis and localization of cusp prolapse associated with ascending aortic disease and may help to improve results of aortic valve-sparing procedures.

(J Thorac Cardiovasc Surg 2011;141:917-25)

Aortic valve (AV)-sparing operations are increasingly being used to treat patients with ascending aortic disease with or without aortic insufficiency (AI). ${ }^{1}$ Inability to recognize and treat concomitant cusp disease is an important reason for immediate and late failure of valve-sparing operations. ${ }^{2}$ As such, significant preoperative AI is often considered a contraindication to valve-sparing operations. The most common form of cusp disease, either pre-existing or induced during valve-sparing surgery, that leads to recurrent $\mathrm{AI}$ and failure of the procedure is cusp prolapse.

\footnotetext{
From the Department of Cardiovascular and Thoracic Surgery, ${ }^{a}$ Cliniques Universitaires Saint-Luc, Brussels, Belgium; and the Division of Cardiac Surgery, ${ }^{\mathrm{b}}$ University of Ottawa Heart Institute, Ottawa, Ontario, Canada.

Disclosures: Authors have nothing to disclose with regard to commercial support.

Received for publication May 4, 2010; revisions received Sept 14, 2010; accepted for publication Dec 4, 2010; available ahead of print Feb 4, 2011.

Read at the 90th Annual Meeting of The American Association for Thoracic Surgery, Toronto, Ontario, Canada, May 1-5, 2010.

Address for reprints: Munir Boodhwani, MD, MMSc, Service de Chirurgie Cardiovasculaire et Thoracique, Cliniques Universitaires Saint-Luc UCL 90, Avenue Hippocrate 10, Brussels B-1200, Belgium (E-mail: mboodhwani@ottawaheart.ca). $0022-5223 / \$ 36.00$

Copyright (c) 2011 by The American Association for Thoracic Surgery doi:10.1016/j.jtcvs.2010.12.006
}

The prevalance of AV cusp prolapse as an isolated lesion in the general population is quite low. ${ }^{3}$ Although it is an imminently repairable lesion, most patients with cusp prolapse as the sole cause of severe $\mathrm{AI}$ undergo $\mathrm{AV}$ replacement. This is due, in large part, to the lack of preoperative and intraoperative recognition of the lesion, insufficient experience with surgical repair techniques, and the lack of long-term data on outcome after repair. A number of surgical techniques have now been described for the repair of cusp prolapse with acceptable midterm to long-term outcomes. ${ }^{4,5}$ However, the clinical, echocardiographic, and intraoperative features of this pathologic entity that would enable accurate diagnosis, assessment, and appropriate treatment have not been systematically described. Furthermore, a better understanding of the salient features of isolated aortic cusp prolapse can potentially provide important insight into the management of cusp prolapse associated with ascending aortic disease and may lead to improved outcomes after $\mathrm{AV}$-sparing operations.

From a large population of unselected patients undergoing nonemergency $\mathrm{AV}$ repair, we identified all patients who underwent repair of aortic cusp prolapse. Patients with bicuspid AVs were excluded because they exhibit significant 


\section{Abbreviations and Acronyms \\ $\mathrm{AI}=$ aortic insufficiency \\ $\mathrm{AV}=$ aortic valve}

anatomic and morphologic variations and have important differences in cusp quality and mechanisms of AI compared with patients with trileaflet AVs. ${ }^{6}$ The specific objectives of this study were as follows:

1. To examine clinical characteristics as well as echocardiographic and intraoperative features of patients who underwent $\mathrm{AV}$ repair for isolated cusp prolapse

2. To compare these characteristics in patients undergoing cusp repair with concomitant ascending aortic dilatation

3. To compare midterm and long-term outcome after AV repair for cusp prolapse, with or without ascending aortic disease

\section{METHODS}

This study was approved by the Ethics Review Board of Cliniques Universitaires Saint-Luc and written informed consent was waived for this study.

\section{Patient Population}

Between March 1996 and September 2009, 428 consecutive patients underwent nonemergency AV repair for AI with or without ascending aortic disease. Of these, 194 (45\%) were treated for cusp prolapse; $111(57 \%)$ of these had trileaflet AVs and make up the study cohort. The overall approach to the treatment of AI was guided by our "Repair-Oriented Functional Classification of Aortic Insufficiency."7 According to this classification, AI associated with normal cusp motion (largely owing to dilatation of the functional aortic annulus) was classified as type 1, AI owing to cusp prolapse was classified as type 2 , and that owing to cusp restriction was classified as class 3 . Study patients were divided into 2 groups: those with isolated cusp prolapse (type 2 disease) in the absence of ascending aortic or aortic root dilatation (isolated group, $\mathrm{n}=50$ ) and those who had cusp prolapse treated in association with ascending aortic dilatation (type 1 disease), either at the level of the supracoronary ascending aorta (type 1a) or the aortic root (type 1b). These patients made up the associated group $(\mathrm{n}=61)$.

Data on surgical techniques were collected prospectively and analyzed retrospectively. Preoperative characteristics according to study group are described in Table 1 . The mean age of the entire cohort was $57 \pm 17$ years and $92 \%$ were male. In this cohort, $12(11 \%)$ patients had had a previous Ross operation and presented with AI with or without dilatation of the pulmonary autograft. Two additional patients had had a previous AV repair at a different center ( 1 for congenital AI associated with a ventricular septal defect).

\section{Definition and Echocardiographic Identification of Cusp Prolapse}

Cusp coaptation in a normal trileaflet $\mathrm{AV}$ occurs at the mid-height of the sinuses of Valsalva. Cusp prolapse was therefore defined as the visualization of an aortic cusp below this physiologic height of coaptation and often prolapsing into the left ventricle during diastole. Single cusp prolapse is typically identifiable in comparison with two adjacent "normal" or non-
TABLE 1. Preoperative data

\begin{tabular}{lccc}
\hline \multicolumn{1}{c}{ Characteristic } & $\begin{array}{c}\text { Isolated } \\
(\mathbf{n = 5 0 )}\end{array}$ & $\begin{array}{c}\text { Associated } \\
(\mathbf{n = 6 1 )}\end{array}$ & $\begin{array}{c}\boldsymbol{P} \\
\text { value }\end{array}$ \\
\hline Age & $57 \pm 16$ & $56 \pm 17$ & .68 \\
Male & $46(92 \%)$ & $57(92 \%)$ & .97 \\
NYHA class & & & \\
$\quad$ I & $13(26 \%)$ & $31(51 \%)$ & .01 \\
II & $27(54 \%)$ & $26(43 \%)$ & \\
III & $10(20 \%)$ & $4(7 \%)$ & \\
LV ejection fraction $<50 \%$ & $12(24 \%)$ & $4(7 \%)$ & .008 \\
Previous cardiac surgery & $6(12 \%)$ & $8(13)$ & .86 \\
LVEDD (mm) & $62 \pm 9$ & $59 \pm 8$ & .05 \\
Aortic size (mm) & - & $51 \pm 7$ & - \\
Concomitant procedures & $23(46 \%)$ & $15(25 \%)$ & .02 \\
$\quad$ CABG & $11(22 \%)$ & $8(13 \%)$ & \\
Mitral valve repair/replacement & $12(24 \%)$ & $6(10 \%)$ & \\
Other & $6(12 \%)$ & $2(3 \%)$ & \\
\hline
\end{tabular}

NYHA, New York Heart Association; $L V$, left ventricle; $L V E D D$, left ventricular enddiastolic diameter; $C A B G$, coronary artery bypass grafting.

prolapsing cusps. Echocardiographic findings in this setting (depicted in Figure 1) include excessive cusp motion, an eccentric AI jet directed in the opposite direction to the prolapsing cusp on the long-axis view of the $\mathrm{AV}$. A short-axis view often identifies the origin of the AI jet along the entire coaptation margin of the prolapsing cusp. This is in contrast to AI owing to annular dilatation, which is typically central in origin. Additional corroborative findings include a reduced length of cusp coaptation as well as the presence of a fibrous band visible on the prolapsing cusp on long- and short-axis views of the AV. The identification of the band also helps to localize the prolapsing cusp, which is most useful when one of the posterior cusps (left coronary or noncoronary) is prolapsing, inasmuch as they are not easily distinguishable on a long-axis view of the AV.

Multiple cusp prolapse is identifiable by careful echocardiographic examination of the motion of each cusp during diastole and is frequently associated with a low height of cusp coaptation. The eccentricity and orientation of the regurgitant jet in this setting may be variable.

\section{Surgical Assessment and Repair Techniques}

After median sternotomy, institution of cardiopulmonary bypass, and cardioplegia, the AV was inspected. The AV was systematically exposed in all cases by making a transverse aortotomy approximately $1 \mathrm{~cm}$ above the sinotubular junction, leaving the posterior 2 to $3 \mathrm{~cm}$ of aortic wall intact. In cases of ascending aortic dilatation, the aorta was transected at this level. The distal aorta was retracted cephalad and 4-0 polypropylene sutures were placed at the level of each commissure.

The AV was assessed by placing axial traction (perpendicular to the annular plane) on the commissural retraction sutures. This maneuver demonstrates physiologic aortic AV closure position and the area and height of coaptation can be observed and the relative heights of the cusps can be compared. A prolapsing cusp can be identified as having excess free margin length and frequently exhibits a transverse fibrous band that is visible on echocardiography as described above as well as on surgical inspection (Figure 2). Next, radial traction (parallel to the annular plane) is applied to the 3 commissural sutures and the center of the cusp free margin can then be gently pushed down toward the left ventricle with a forceps. A nonprolapsing cusp will remain at its physiologic coaptation level (halfway between the base of the cusp and its maximal height at the commissure) whereas a prolapsing cusp will be able to be pushed lower owing to excessive cusp tissue.

Once the prolapsing cusp(s) had been identified, cusp repair was performed using free margin plication, free margin resuspension using 7-0 

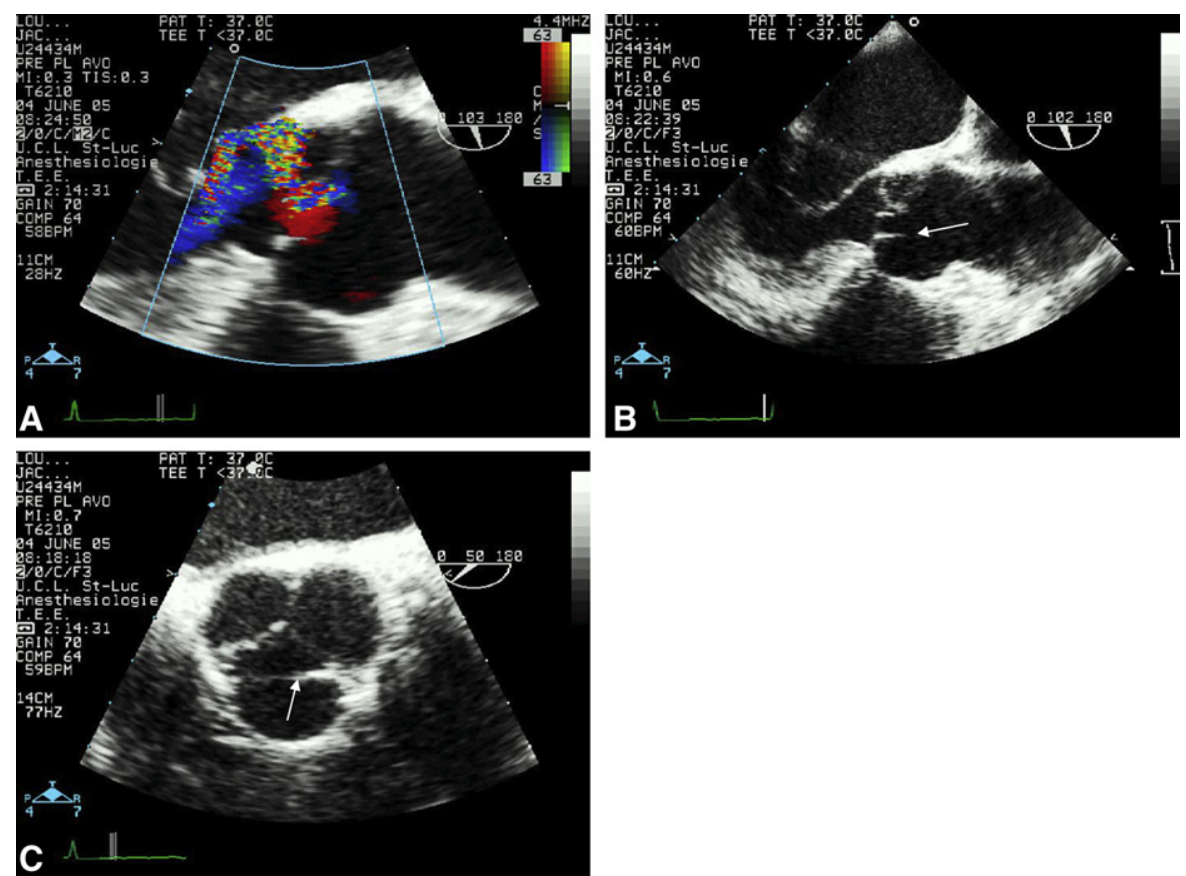

FIGURE 1. Transesophageal echocardiographic views of the aortic valve in long axis (A), demonstrating an eccentric aortic insufficiency jet. B, Cusp prolapse with coaptation below the level of the aortic annulus and a fibrous band (white arrow). C, A short-axis view of the aortic valve confirms the presence of the fibrous band (white arrow).
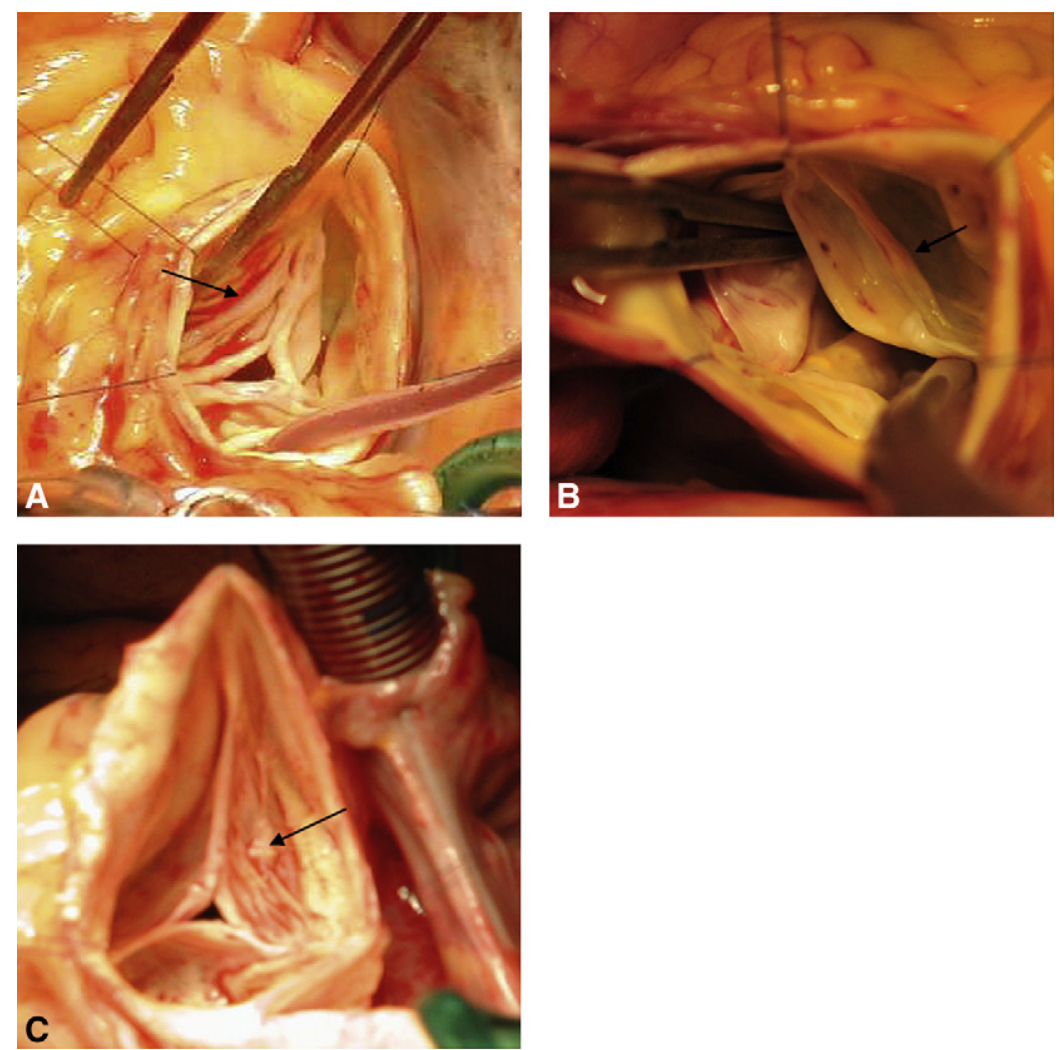

FIGURE 2. After a transverse aortotomy, cusp inspection reveals a transverse fibrous band on the prolapsing cusp indicated by the black arrow. Right coronary cusp (A and B) and noncoronary cusp (C). 
TABLE 2. Operative data

\begin{tabular}{|c|c|c|c|}
\hline Characteristic & $\begin{array}{l}\text { Isolated } \\
(\mathbf{n}=\mathbf{5 0})\end{array}$ & $\begin{array}{c}\text { Associated } \\
(\mathrm{n}=61)\end{array}$ & $\begin{array}{c}P \\
\text { value }\end{array}$ \\
\hline Cardiac ischemia time (min) & $72 \pm 33$ & $113 \pm 31$ & $<.001$ \\
\hline Cardiopulmonary bypass time (min) & $86 \pm 32$ & $125 \pm 30$ & $<.001$ \\
\hline No. of cusps repaired & & & .27 \\
\hline One cusp & $36(72 \%)$ & $47(77 \%)$ & \\
\hline Two cusps & $13(26 \%)$ & $10(16 \%)$ & \\
\hline Three cusps & $1(2 \%)$ & $4(7 \%)$ & \\
\hline \multicolumn{4}{|l|}{ Cusps repaired } \\
\hline Right coronary cusp & $35(70 \%)$ & $40(66 \%)$ & .62 \\
\hline Noncoronary cusp & $21(42 \%)$ & $17(28 \%)$ & .12 \\
\hline Left coronary cusp & $9(18 \%)$ & $22(36 \%)$ & .03 \\
\hline Cusp repair technique & & & .48 \\
\hline Free margin plication & $18(37 \%)$ & $29(48 \%)$ & \\
\hline Free margin resuspension & $16(33 \%)$ & $19(32 \%)$ & \\
\hline Plication + resuspension & $11(22 \%)$ & $10(17 \%)$ & \\
\hline $\begin{array}{l}\text { Triangular resection } \pm \text { other } \\
\text { techniques }\end{array}$ & $4(8 \%)$ & $2(3 \%)$ & \\
\hline Aortic annulus interventions & & & - \\
\hline Subcommissural annuloplasty & $46(92 \%)$ & $13(21 \%)$ & \\
\hline \multicolumn{4}{|l|}{ Valve-sparing root replacement } \\
\hline Reimplantation technique & - & $49(80 \%)$ & \\
\hline Remodeling technique & - & $2(3 \%)$ & \\
\hline Supracoronary aortic replacement & - & $11(18 \%)$ & \\
\hline
\end{tabular}

polytetrafluoroethylene suture, or both as previously described. ${ }^{4,8}$ In a few patients $(n=6)$, a triangular resection was performed in the central portion of the cusp to resect thickened and fibrotic cusp tissue. A total of 144 cusps were repaired in 111 patients (1.3 cusps/patient) using 198 repair techniques (1.4 techniques/cusp). The most commonly repaired cusp was the right coronary cusp $(\mathrm{n}=75)$, followed by the noncoronary cusp $(n=38)$ and the left coronary cusp $(n=31)$. The number of cusps repaired in the isolated versus associated groups and the cusp repair techniques used along with other intraoperative data are depicted in Table 2.

Annular stabilization was performed systematically using subcommissural annuloplasty in the isolated group and in those patients in the associated group who underwent supracoronary ascending aortic replacement. Subcommissural annuloplasty was performed using braided sutures. The first arm of the suture was passed from the aortic to the ventricular side, in the interleaflet triangle, coming back out to the aortic side at the same level. The second arm of the suture was passed in a similar fashion just below the first. A free pledget was added and the suture was tied. This maneuver helps to stabilize the ventriculoaortic junction, reduces the width of the interleaflet triangles, and increases the coaptation surface of the valve leaflets. In the rest of the patients in the associated group, valve-sparing root replacement was performed preferentially using the reimplantation technique as previously described. All patients underwent intraoperative, postrepair echocardiography to assess the degree of residual $\mathrm{AI}$ and the orientation of the regurgitant jet (if present), as well as the coaptation length and coaptation height of the AV cusps. Absence of AI, a coaptation length of at least $5 \mathrm{~mm}$ at the midportion of the free margin, and a coaptation height above the $\mathrm{AV}$ annulus were criteria for a successful repair. ${ }^{9}$

\section{Echocardiographic Review}

All preoperative and intraoperative echocardiograms were reviewed by a trained echocardiographer blinded to intraoperative findings and surgical techniques used. In particular, data on the severity, origin, and eccentricity of the AI jet were collected along with the preoperative echocardiographic diagnosis of cusp prolapse. The presence or absence of the fibrous band and its location was noted. Last, the dimensions of the ascending aorta were measured at various levels.

\section{Follow-up}

Clinical follow-up was conducted through either outpatient visits or telephone follow-up by a research nurse. Information on survival status and valve-related complications, including thromboembolism, hemorrhage, endocarditis, reoperation, and cardiovascular symptoms, was obtained. Transthoracic echocardiography was obtained on all patients before discharge and at regular intervals during follow-up. The closing interval for the study was between February 2010 and April 2010. Clinical follow-up was $100 \%$ complete with a mean follow-up time of 3.8 years (range, 0.1-13.3 years). Echocardiographic follow-up was 100\% complete at a mean follow-up time of 3.2 years (range, $0.1-11.9$ years).

\section{Statistical Analysis}

Valve-related outcomes were defined as per published guidelines. ${ }^{10}$ Continuous data are presented as mean \pm standard deviation or median (interquartile range) for nonparametric data. Failure time data on survival, reoperation, and recurrent AI are presented using Kaplan-Meier survival curves. Comparisons between groups for failure time data were performed using the $\log$ rank test. Statistical analyses were performed using SAS version 9.2 (SAS Institute, Inc, Cary, NC). Graphs were constructed using GraphPad Prism 5.0 (GraphPad Software, Inc, San Diego, Calif).

\section{RESULTS}

\section{Clinical Characteristics}

Preoperative patient characteristics are presented in Table 1 . Of the 50 patients in the isolated group, $4(8 \%)$ had previously undergone a Ross procedure and had AI owing to cusp prolapse in the pulmonary autograft. Three of these 4 patients were treated for multiple cusp prolapse. The majority of the patients in the isolated group were symptomatic (New York Heart Association class II or greater) and about one fourth showed signs of left ventricular dysfunction. Ten (20\%) patients in this group had moderate AI owing to cusp prolapse while undergoing other concomitant cardiac procedures.

In contrast, of the 61 patients in the associated group, 8 $(13 \%)$ had previously had a Ross procedure and presented with pulmonary autograft dilatation associated with cusp prolapse and AI. In all these patients, only single cusp prolapse repair was performed. The majority of the patients in this group were asymptomatic, and aortic dilatation with associated AI was the primary indication for operation. Ten $(16 \%)$ patients in this group had only mild preoperative AI but were found to have cusp prolapse after correction of their aortic disease. Mean aortic diameter was $51 \mathrm{~mm}$ and $7(11 \%)$ had a diagnosis of Marfan syndrome.

\section{Echocardiographic Features}

Patients with isolated AI had more severe insufficiency (Table 3; $P=.005$ ) and larger left ventricular diameters. An eccentric AI jet was found in $83 \%$ of patients in the isolated group and $63 \%$ of the associated group $(P=.02)$. A 
TABLE 3. Echocardiographic features

\begin{tabular}{lccc}
\hline Characteristic & Isolated $(\mathbf{n = 4 8 )}$ & Associated $(\mathbf{n}=\mathbf{5 4})$ & $\boldsymbol{P}$ value \\
\hline AI severity & & & \\
$1+$ & 0 & $10(16 \%)$ & .005 \\
$2+$ & $10(20 \%)$ & $16(26 \%)$ & \\
$3+$ or $4+$ & $40(80 \%)$ & $35(57 \%)$ & \\
Eccentric jet & $40(83 \%)$ & $34(63 \%)$ & .02 \\
Band & $32(67 \%)$ & $20(34 \%)$ & .001 \\
Cusp prolapse & $41(85 \%)$ & $38(66 \%)$ & .02 \\
\hline
\end{tabular}

fibrous band was identified in $67 \%$ of patients in the isolated group and $34 \%$ of the associated group $(P=.001)$. A diagnosis of cusp prolapse on preoperative echocardiography could be made in $85 \%$ of the isolated group and $66 \%$ of the associated group. Overall, the presence of an eccentric AI jet, regardless of severity, had a sensitivity and specificity of $92 \%$ and $96 \%$, respectively. The presence of a fibrous band had an overall sensitivity and specificity of $57 \%$ and $92 \%$ for the echocardiographic diagnosis of cusp prolapse in the setting of significant $\mathrm{AI}(>2+)$.

\section{Clinical Outcome}

There was no in-hospital mortality and no patients required early $\mathrm{AV}$ reintervention. Four patients required a permanent pacemaker postoperatively, and 10 required re-exploration for postoperative bleeding. Intraoperative postrepair echocardiography revealed none or mild ( 0 or $1+) \mathrm{AI}$ in $105(95 \%)$ patients and $2+\mathrm{AI}$ in $6(5 \%)$ patients. At discharge echocardiography, $13(12 \%)$ were found to have $2+\mathrm{AI}$ with the rest $(88 \%)$ ) having either 0 or $1+\mathrm{AI}$.

During the follow-up period, 5 patients died ( 1 in the isolated group, 4 in the associated group), of whom 2 died of cardiac causes. Although there were no documented valve-related deaths, 1 patient died of a presumed ventricular arrhythmia. Four (4\%) patients underwent AV reoperation (1 in the isolated group and 3 in the associated group). Two patients had recurrent AI, 1 with mixed AV stenosis and insufficiency, and 1 had with a fistula from the aorta to the right atrium. At 8 years, freedom from $\mathrm{AV}$ reoperation was $100 \%$ in the isolated group and $93 \% \pm 4 \%$ in the associated group $(P=.33)$. The single reoperation in the isolated group occurred just after 8 years. Freedom from recurrent $\mathrm{AI}(>2+)$ was $87 \% \pm 7 \%$ in the isolated group and $93 \% \pm 5 \%$ in the associated group (Figure 3). Valverelated complications included thromboembolic complications in 2 patients (transient ischemic attack in a patient with a history of atrial fibrillation and stroke in the other patient) and $4 \mathrm{AV}$ reoperations as described earlier. No patients had endocarditis or bleeding events during the follow-up period. Overall freedom from thromboembolism, bleeding, or endocarditis was $98 \% \pm 2 \%$ at 8 years.

Subgroup analyses revealed that the surgical technique (plication vs resuspension vs both; $P=.6$ ) or the number
Freedom from AV Reoperation
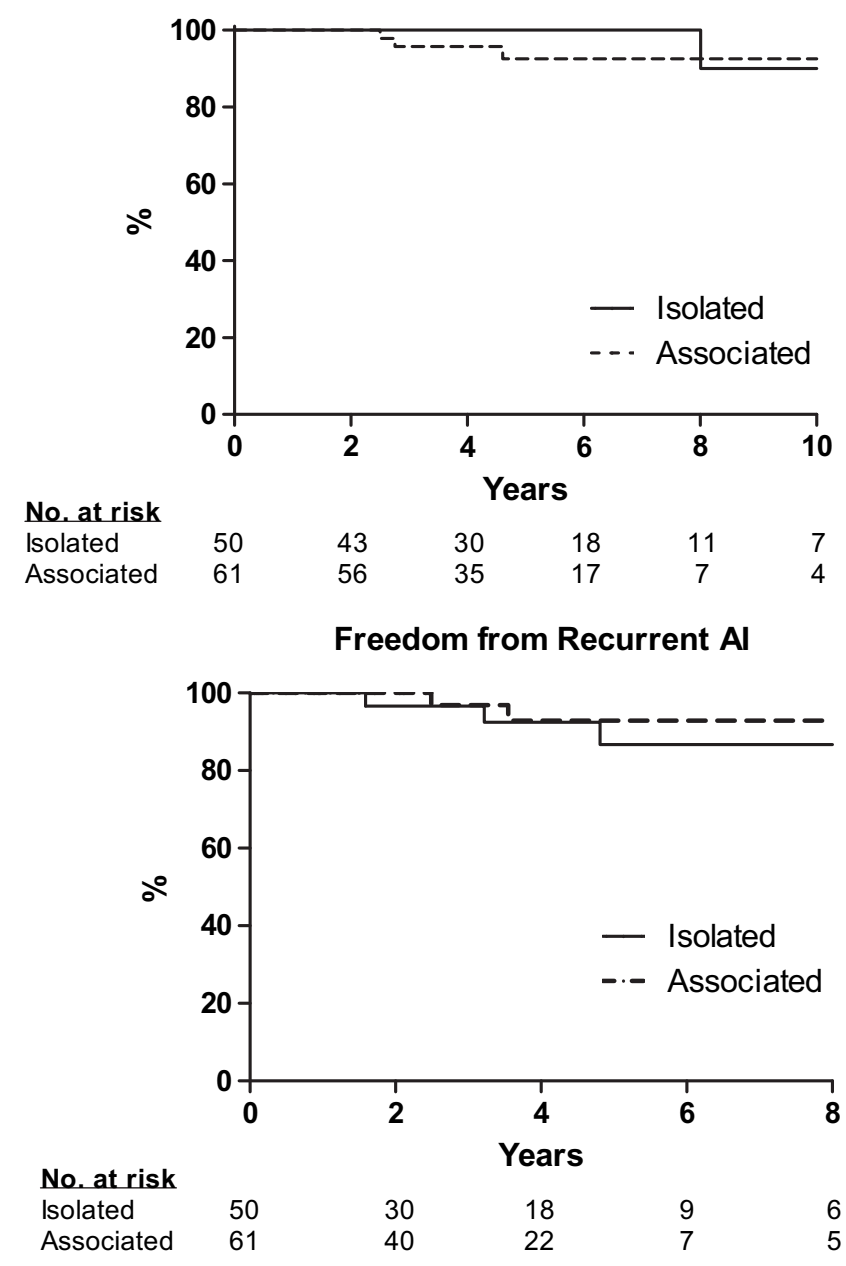

FIGURE 3. Freedom from (A) aortic valve $(A V)$ reoperation and (B) recurrent aortic insufficiency $(A I)$ greater than $2+$ in the isolated and associated groups.

of cusps repaired (single vs multiple; $P=.39$ ) had no effect of freedom from recurrent AI at follow-up.

\section{DISCUSSION}

Cusp prolapse is an infrequent cause of isolated $\mathrm{AI}$ in trileaflet valves. However, diagnosis, assessment, and repair of cusp prolapse is an integral component of AV repair and $\mathrm{AV}$-sparing surgery. In this article, we report that echocardiographic findings, such as an eccentric AI jet, are highly sensitive and specific $(92 \%$ and $96 \%)$ for the diagnosis of prolapse, and the presence of a fibrous band, on echocardiography and intraoperative examination, is very specific $(92 \%)$ and can help to localize the prolapsing cusp. Cusp prolapse most frequently involves the right coronary cusp, followed by the noncoronary and then the left coronary cusp, which is infrequently involved in isolated cusp disease. The majority of patients $(\sim 75 \%)$, both in 
the isolated and associated groups, require single cusp repair but the rest have multiple cusp involvement. At follow-up, cusp repair provides a high freedom from $\mathrm{AV}$ reoperation, both in the isolated (100\% at 8 years) and the associated group (93\% at 8 years), and recurrent AI occurs at acceptable rates. These results are independent of the repair technique used. The incidence of valve-related complications is low in both groups. In summary, careful echocardiographic and intraoperative evaluation provides important clues for the evaluation and repair of cusp prolapse with or without ascending aortic disease.

Cusp prolapse, as an isolated cause of AI, is a relatively uncommon entity. In a cross-sectional study of 2000 patients undergoing echocardiography, Shapiro and associates $^{3}$ found cusp prolapse in $1.2 \%$. It was even less common when restricted to patients with trileaflet AVs and those without ascending aortic dilatation. As such, the clinical and echocardiographic characteristics of patients with this pathologic entity have not been well described. In 50 patients with isolated cusp prolapse (46 native $\mathrm{AVs}$, 4 pulmonary autografts) as the primary cause of AI, the mean age was relatively young at 57 years. The vast majority $(83 \%)$ had an eccentric AI jet and a fibrous band was discernible in $66 \%$ on echocardiography. Cohen and associates ${ }^{11}$ also examined this issue in a mixed population of patients with $\mathrm{AI}$ and found that $69 \%$ of patients with an eccentric AI jet had cusp prolapse. Most patients in our cohort had single cusp involvement and only 1 patient, with a previous Ross procedure, required repair of all 3 cusps of a pulmonary autograft. The etiology and pathophysiology of isolated cusp prolapse are unclear and are likely degenerative in nature, perhaps owing to longstanding hypertension. Consistent with previous reports, however, $12(24 \%)$ of these patients also had associated mitral valve disease requiring mitral valve repair, suggesting perhaps an intrinsic abnormality of valvular cusp tissue. ${ }^{3}$

The management of AV cusp prolapse is of critical importance during the conduct of $\mathrm{AV}$-sparing operations. Cusp prolapse in this setting may be pre-existing, may be unmasked after the correction of aortic root dimensions, or may be induced owing to technical errors during the valve-sparing procedure. For the surgeon performing an $\mathrm{AV}$-sparing root replacement, several important lessons can be learned from the findings of this study. First, if the dilatation of the ascending aorta is accompanied by an eccentric AI jet, single cusp prolapse should be suspected. This is true even if the AI is mild in severity. Eccentric AI is clearly a hallmark of single cusp prolapse, and this finding alone identifies a majority of patients requiring cusp repair (63\% of the associated group). Second, if cusp prolapse exists, it is most likely to involve the right coronary cusp $(\sim 75 \%)$. A posteriorly directed AI jet is characteristically found in these cases. Third, if a transverse fibrous band is detected on echocardiography and intraoperative inspec- tion, this finding is very specific ( $93 \%$ specificity) and can help to localize the cusp prolapse. However, the sensitivity of this finding is low; that is, the lack of a fibrous band does not rule out the need for cusp repair. Fourth, patients with mild preoperative AI are unlikely to require cusp repair. In our cohort, only $16 \%$ of patients requiring cusp repair in the associated group had mild (1+) AI. Fifth, in cases in which residual AI is found after a valve-sparing root replacement procedure, the presence of an eccentric AI jet suggests uncorrected cusp prolapse that can be localized and corrected. Last, there is a subset of patients, particularly those with induced cusp prolapse, in whom the need for cusp repair cannot be predicted by preoperative echocardiography, and this underscores the need for a thorough intraoperative assessment of the AV.

In our view, this assessment can be optimally performed just before the distal aortic anastomosis, that is, after the proximal portion of the valve-sparing root replacement procedure and reimplantation of the coronary buttons is complete. A dose of cardioplegic solution is given through the neoaortic root, which allows distention of the aortic root to physiologic pressures. In addition to assessing hemostasis at the coronary buttons, a limited echocardiographic view may be obtained at this time to look for significant AI. At the end of cardioplegia delivery, the residual fluid is aspirated from the aortic root without disturbing the valve cusps and traction is applied to the 3 commisures. This gives a view of the $\mathrm{AV}$ in its physiologic closed position. Cusp prolapse identified at this time can then be corrected by previously described techniques.

Several techniques have been described to repair aortic cusp prolapse. In trileaflet AVs, where the cusp tissue is typically thin and pliable, central free margin plication and free margin resuspension are the most common techniques used. Less commonly, trileaflet AV cusps may contain thickened and fibrotic portions that require a parsimonious triangular resection with either primary closure or cusp restoration with a pericardial patch. Free margin plication is quick and easy to perform and is applicable in most situations, whereas free margin resuspension is useful to close coexisting stress fenestrations and to homogenize the free margin when a pericardial patch is used. These techniques can be combined and appear to provide similar durability, as has been reported by our group and others. ${ }^{12,13}$ Thus, the choice of technique can be tailored to the pathologic condition encountered.

The conventional treatment of adult patients with aortic cusp prolapse and significant AI has been AV replacement. Given the young age of this patient cohort (mean age 57 years), many of these patients would undergo mechanical AV replacement. This study demonstrates that AV repair is feasible in this patient population and can be performed safely with low rates of $\mathrm{AV}$ reoperation and acceptable rates of recurrent AI. The combined rate of valve-related 
complications of thromboembolism, bleeding, and endocarditis are quite low at $0.47 \%$ /person-years. This is consistent with previous reports ${ }^{14}$ and is significantly lower than the $1 \%$ to $2 \%$ /patient-years incidence of thromboembolic complications in patients receiving prosthetic valves. ${ }^{15,16}$ In addition, mechanical heart valves have the added risk and inconvenience of anticoagulation-related complications. However, longer-term follow-up in larger patient cohorts is necessary to truly establish the role of $\mathrm{AV}$ repair in patients with cusp prolapse.

\section{LIMITATIONS}

This is a single center study in which techniques and indications for $\mathrm{AV}$ repair have evolved over the past 15 years. Echocardiographic review, although blinded, was performed in a retrospective manner. Furthermore, not all of the follow-up echocardiographic studies were performed at the authors' institution and some lacked important quantitative and qualitative parameters that could give important insight into mechanisms of repair failure and recurrent AI.

\section{CONCLUSIONS}

Isolated cusp prolapse causing $\mathrm{AI}$ is an uncommon entity that is amenable to valve repair. Careful echocardiographic and intraoperative identification of cusp prolapse is critical for successful repair for both isolated lesions as well as those associated with ascending aortic disease. Important lessons from the study of isolated cusp prolapse can be translated to patients having $\mathrm{AV}$-sparing operations. Outcome after AV repair in this setting is durable in the mid term and is associated with a low incidence of valverelated complications.

\section{References}

1. David TE, Feindel CM, Webb GD, Colman JM, Armstrong S, Maganti M. Longterm results of aortic valve-sparing operations for aortic root aneurysm. J Thorac Cardiovasc Surg. 2006;132:347-54.

2. Burkhart HM, Zehr KJ, Schaff HV, Daly RC, Dearani JA, Orszulak TA. Valve preserving aortic root reconstruction: a comparison of techniques. J Heart Valve Dis. 2003;12:62-7.

3. Shapiro LM, Thwaites B, Westgate C, Donaldson R. Prevalence and clinical significance of aortic valve prolapse. Br Heart J. 1985;54:179-83.

4. Boodhwani M, de Kerchove L, Glineur D, El Khoury G. A simple method for the quantification and correction of aortic cusp prolapse by means of free margin plication. J Thorac Cardiovasc Surg. 2010;139:1075-7.

5. David TE, Armstrong S. Aortic cusp repair with Gore-Tex sutures during aortic valve-sparing operations. J Thorac Cardiovasc Surg. 2010;139:1340-2.

6. Boodhwani M, de Kerchove L, Glineur D, Rubay J, Vanoverschelde JL, Noirhomme P, et al. Repair of regurgitant bicuspid aortic valves: a systematic approach. J Thorac Cardiovasc Surg. 2010;140:276-84.

7. Boodhwani M, de Kerchove L, Glineur D, Poncelet A, Rubay J, Astarci P, et al. Repair-oriented classification of aortic insufficiency: impact on surgical techniques and clinical outcomes. J Thorac Cardiovasc Surg. 2009;137:286-94.

8. El Khoury G, Vanoverschelde JL, Glineur D, Poncelet A, Verhelst R, Astarci P, et al. Repair of aortic valve prolapse: experience with 44 patients. Eur J Cardiothorac Surg. 2004;26:628-33.

9. le Polain de Waroux JB, Pouleur AC, Robert A, Pasquet A, Gerber BL, Noirhomme $\mathrm{P}$, et al. Mechanisms of recurrent aortic regurgitation after aortic valve repair: predictive value of intraoperative transesophageal echocardiography. JACC Cardiovasc Imaging. 2009;2:931-9.
10. Akins CW, Miller DC, Turina MI, Kouchoukos NT, Blackstone EH, Grunkemeier GL, et al. Guidelines for reporting mortality and morbidity after cardiac valve interventions. J Thorac Cardiovasc Surg. 2008;135:732-8.

11. Cohen GI, Duffy CI, Klein AL, Miller DP, Cosgrove DM, Stewart WJ. Color Doppler and two-dimensional echocardiographic determination of the mechanism of aortic regurgitation with surgical correlation. J Am Soc Echocardiogr. 1996;9:508-15.

12. Aicher D, Langer F, Adam O, Tscholl D, Lausberg H, Schafers HJ. Cusp repair in aortic valve reconstruction: does the technique affect stability? $J$ Thorac Cardiovasc Surg. 2007;134:1533-8; discussion 1538-9.

13. de Kerchove L, Glineur D, Poncelet A, Boodhwani M, Rubay J, Dhoore W, et al Repair of aortic leaflet prolapse: a ten-year experience. Eur J Cardiothorac Surg. 2008;34:785-91.

14. Aicher D, Fries R, Rodionycheva S, Schmidt K, Langer F, Schafers HJ. Aortic valve repair leads to a low incidence of valve-related complications. Eur J Cardiothorac Surg. 2010;37:127-32.

15. Peterseim DS, Cen YY, Cheruvu S, Landolfo K, Bashore TM, Lowe JE, et al Long-term outcome after biologic versus mechanical aortic valve replacement in 841 patients. J Thorac Cardiovasc Surg. 1999;117:890-7.

16. Ruel M, Masters RG, Rubens FD, Bedard PJ, Pipe AL, Goldstein WG, et al. Late incidence and determinants of stroke after aortic and mitral valve replacement. Ann Thorac Surg. 2004;78:77-83; discussion 84.

\section{Discussion}

Dr Marc R. Moon (St Louis, Mo). Dr Boodhwani, I would like to congratulate you and your coauthors on achieving a very systematic approach and developing an excellent program in AV repair. These are very challenging patients historically to treat. I do have a few questions and comments that I think may help clarify some of your presentation.

During this period, did you also have a set of patients with cusp prolapse on whom you performed AV replacement? If so, how did you determine who needed a replacement and who underwent a repair? For example, in patients who had some calcium on a leaflet, which is very common, obviously, in elderly patients, did you debride the calcium and then perform a leaflet repair or did those patients all get a valve replacement?

In your manuscript you discuss performing a limited echocardiographic evaluation of the valve before closing the aorta. This is done after completing the proximal portion of a Tirone David procedure, for example, and then insufflating the root and doing an echocardiogram. I think that my anesthesiologist may have a difficult time getting those images, especially when the left ventricle is empty. How often was your anesthesiologist able to get those images, were they most often successful, and were there any tricks or techniques you can recommend to improve the yield?

Your study group also consisted of only patients with tricuspid valve disease. You reported during that same time period you had over 80 bicuspid valves. Without knowing all the exact numbers, can you give us an impression as to whether you were able to achieve equal success with those patients as well.

Finally, in the abstract and your presentation and manuscript you refer to the 8 -year freedom from reoperation rate as $100 \%$ in the isolated and $93 \%$ in the associated group. From your presentation, we would have thought you achieved perfection with this procedure in the isolated group at least. It is my belief that in the presentation it might have been a little more fair to present your 10-year rate, which probably approximated 90\%-still an excellent result but I think probably better representing your data.

Dr Boodhwani. Thank you, Dr Moon. Those are very insightful comments. 
In general, AV repair is reserved for and will likely have the greatest benefit in younger patients who would typically undergo otherwise a mechanical AV replacement and have the cumulative burden of the valve-related morbidity that occurs with that. Thus elderly patients, particularly those in whom cusp quality is not adequate, are not typically candidates for AV repair. Having said that, this series also represents a 13-year experience and over that experience the practice has invariably evolved. It has evolved now to the point that most patients who have isolated AI are considered a priori as being candidates for repair. If intraoperatively or on the pre-repair transesophageal echocardiogram we find aspects of the valve that would not be amenable to repair, then those valves are replaced, because we have really quite good prostheses available and good results with valve replacement.

The second question was regarding the limited echocardiographic view. The one adjunctive maneuver that can be done to try to fill up the heart a little bit more is to clamp the left ventricular vent. However, frankly, that is a difficult view to obtain and it has taken a lot of practice for the echocardiographers at our institution to actually obtain that view. Often it is not a complete view of the valve, mind you, but it is a view looking at the long axis of the AV as it goes into the left ventricular outflow tract. You are looking for major jets of AI, and that gives you a clue as to whether your repair is going to be a complete failure or whether it is going to be something acceptable. It is not a complete echocardiographic assessment by any means.

The third comment was regarding the tricuspid versus bicuspid issue. We actually presented the results of our bicuspid AV repair last year at this meeting and the article is currently in press. The reason we did not include this group in this particular setting is that bicuspid AVs often present with mixed disease. There is some cusp restriction often owing to restrictive raphe along with prolapse, and it would not be fair to include those valves in the same category as these valves. Certainly another important difference is that the techniques used for bicuspid AVs are quite significantly different; because a lot of thickened, sometimes calcified, raphe tissue needs to be excised, sometimes a pericardial patch needs to be used to restore adequate coaptation surface. For that reason we decided to keep this population separate.

Last, I agree with you entirely about the freedom from reoperation at 8 years. The reason we did not include 10-year data is that there were actually very few patients left at the 10-year mark. As a general rule, we truncated our reported follow-up rate at a point where we had at least $10 \%$ to $15 \%$ of the cohort still being censored. That is the reason that the 8 -year follow-up appears perhaps a little overstated.

Dr Robert J. Cusimano (Toronto, Ontario, Canada). I am curious about the reasons for failure later on. Was it the same leaflet that you fixed that failed or different leaflets?

Dr Boodhwani. Good question. There were 4 reoperations in the series. One occurred because of mixed stenosis and insufficiency. One occurred because of moderate AI but an aorta-right ventricular fistula that was found during follow-up; this may have been related to a subcommissural annuloplasty suture, and that was the second patient who underwent reoperation. The 2 additional patients who underwent reoperation were both treated for AI, but we did not have documentation of whether it was a repaired cusp that was again prolapsing. I am afraid I cannot answer that.
Dr Cusimano. Do you know by echocardiography?

Dr Boodhwani. I do not have that information, unfortunately.

Dr Yutaka Okita (Kobe, Japan). Regarding size of the aorta, sometimes I believe it is easier to replace the whole root, even in mild dilatation. What was the mean diameter size of the isolated group or Valsalva size, and what was the annular size of the other associated group?

Dr Boodhwani. In the associated group, the question is a little easier to answer. The maximum diameter of the aorta was 51 $\mathrm{mm}$. In the isolated group, not all patients underwent systematic imaging to assess the size of the aorta at the various points. Suffice it to say that our approach in general for replacement of the aortic root is, if the aortic root is definitely greater than $4.5 \mathrm{~cm}$, it is replaced. If it is less than 4.5 but there is significant thinning of the aortic root tissue, then we also take an aggressive approach and replace it.

Again, I want to emphasize one more time that one of the other reasons to replace the root in this setting, other than the complications of dissection and rupture that we all worry about, is to stabilize the aortic annulus in the setting of a repair. This is more true in the bicuspid than in the trileaflet valve, but the bicuspid annuli tend to dilate over time and the stabilization is more secure with a root replacement procedure.

Dr Bansi Koul (Lund, Sweden). I would like to congratulate you for an excellent presentation. We are grateful to your group for providing us continually with the technical knowledge of $\mathrm{AV}$ repairs. We have learned a lot from your group.

One of the problems that I have encountered in tricuspid valve repairs is when more than 1 cusp is engaged. You made a brief remark that the remaining third nonprolapsed cusp remains the reference cusp for repair of the other 2. Could you comment a little more on this, because nonadjoining halves of the 2 prolapsing cusps lack a reference cusp. How do you decide about cusp repair in this situation?

Dr Boodhwani. That is a very good question. What is done in that situation is that the 7-0 Prolene polypropylene that is used to pass through the 2 reference cusps is passed through a single reference cusp, and we start with 1 prolapsing cusp. You pull the prolapsing cusp along the margin of the reference cusp and you take your 6-0 Prolene polypropylene by going from the aortic to the ventricular side, and then you take advantage of symmetry. For example, if you see the nodule of Arantius and you have taken your first bite about $2 \mathrm{~mm}$ on one side, you are going to take your bite coming out again $2 \mathrm{~mm}$ from the center. Once you have done that maneuver, you have 2 corrected cusps and then you can correct the third cusp as was described earlier. Particularly when 3 cusps are prolapsing, this is a real challenge, and this is where it becomes more of an art than a science and requires some eyeballing.

Dr Henning F. Lausberg (Trier, Germany). I congratulate you on excellent results. You are probably aware that a couple of years ago the Homburg group from Germany introduced effective height as a point of reference. This is a little more of a measuring procedure than is eyeballing the relative length of the cusps. Do you use any of those techniques? Right now, measuring devices are also commercially available. Especially if you are doing some root replacement procedures, you might induce some kind of symmetric prolapse. I would like to get your explanation on how you deal with that. 
Dr Boodhwani. I think that is a very good question. We are very familiar with the work of the Homberg group who have come up with a device to measure effective cusp height. There are advantages and disadvantages to it. It is a useful adjunct in AV repair surgery. If you achieve a minimum effective cusp height greater than 8 $\mathrm{mm}$, you have largely restored reasonable coaptation surface. One caveat, however, is that often eccentric AI is not due to absolute cusp prolapse but due to relative prolapse. It is not just the absolute effective height that would be measured by an instrument like this that is important, but the relative height compared with the other cusps. So that is one caveat that I would offer, but that is an acceptable way of assessing appropriate correction of cusp prolapse.

Access to The Journal of Thoracic and Cardiovascular Surgery Online is reserved for print subscribers!

Full-text access to The Journal of Thoracic and Cardiovascular Surgery Online is available for all print subscribers. To activate your individual online subscription, please visit The Journal of Thoracic and Cardiovascular Surgery Online, point your browser to http://www.mosby.com/jtcvs, follow the prompts to activate your online access, and follow the instructions. To activate your account, you will need your subscriber account number, which you can find on your mailing label (note: the number of digits in your subscriber account number varies from 6 to 10$)$. See the example below in which the subscriber account number has been circled:

\section{Sample mailing label}

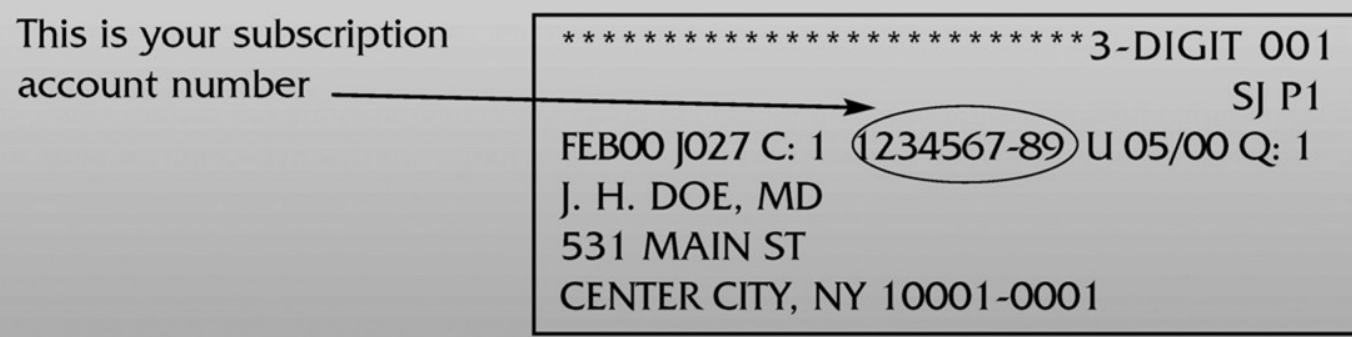

Personal subscriptions to The Journal of Thoracic and Cardiovascular Surgery Online are for individual use only and may not be transferred. Use of The Journal of Thoracic and Cardiovascular Surgery Online is subject to agreement to the terms and conditions as indicated online. 